\title{
Stochastic rotation dynamics. I. Formalism, Galilean invariance, and Green-Kubo relations
}

\author{
T. Ihle $\mathrm{e}^{1,2, *}$ and D. M. Kroll ${ }^{1}$ \\ ${ }^{1}$ Supercomputing Institute, University of Minnesota, 599 Walter Library, 117 Pleasant Street S.E., Minneapolis, Minnesota 55455, USA \\ ${ }^{2}$ IFF, Theorie II, Forschungszentrum Jülich, 52425 Jülich, Germany
}

(Received 27 November 2002; published 12 June 2003)

\begin{abstract}
A detailed analytical and numerical analysis of a recently introduced stochastic model for fluid dynamics with continuous velocities and efficient multi-particle collisions is presented. It is shown how full Galilean invariance can be achieved for arbitrary Mach numbers and how other low temperature anomalies can be removed. The relaxation towards thermal equilibrium is investigated numerically, and the relaxation time is measured. Equations of motions for the correlation functions of coarse-grained hydrodynamic variables are derived using a discrete-time projection operator technique, and the Green-Kubo relations for all relevant transport coefficients are given. In the following paper (Part 2), analytic expressions for the transport coefficients are derived and compared with simulation results. Long-time tails in the velocity and stress autocorrelation functions are measured and shown to be in good agreement with previous mode-coupling theories for continuous systems.
\end{abstract}

DOI: 10.1103/PhysRevE.67.066705

PACS number(s): 02.70.Ns, 47.11.+j, 05.40.-a

\section{INTRODUCTION}

Particle-based simulation techniques have recently emerged as an interesting alternative to more traditional methods for studying such diverse behavior as rarefied gasdynamics, flow and transport in complex geometries and nanometer devices, and the dynamics and rheology of complex liquids such as amphiphilic mixtures, polymer solutions, and colloidal suspensions.

Phenomena involving rarefied gas dynamics and hypersonic flow often occur in regimes where the continuum approximation breaks down and the traditional Navier-Stokes equations are not valid. This is the case, for example, with flows in geometries in which the Knudsen number-defined as the ratio of the mean free path of a gas to the local length scale of the problem-is not negligibly small. If the Knudsen number is larger than $\approx 0.1$, the Navier-Stokes equations are no longer valid, and particle-based methods, such as the direct simulation Monte Carlo (DSMC) [1,2], have been used extensively.

Complex fluids present a challenge for conventional simulation techniques due to the importance of thermal fluctuations and the presence of disparate time scales in their dynamics. On the microscale, molecular dynamics (MD) techniques can be used to model the dynamics of small atomistic systems on time scales ranging from picoseconds to microseconds. However, the slower millisecond dynamics of larger structures, such as the self-assembly of micelles or ordered surfactant phases, cannot be accessed by atomistic methods now or in the foreseeable future. Similarly, although the accurate simulation of the atomic-scale properties of surfactant assemblies and polymers requires atomistic MD simulations, these methods are not suited for determining larger structures such as micellar sizes and shapes, bicontinuous phases, or to predict the phase diagrams of surfactants in

\footnotetext{
*Present address: Institut für Computeranwendungen 1, Universität Stuttgart, Pfaffenwaldring 27, 70569 Stuttgart, Germany.
}

oil or water. At the other extreme, approaches based on the numerical solution of continuum equations encounter difficulties for even such comparatively simple problems as colloidal suspensions due to the cumbersome treatment of moving boundary conditions. In fact, flows with dynamic interfaces are among the most difficult computational problems in continuum mechanics. The central challenges are to develop numerical algorithms that accurately couple the fluid and solid domains and resolve the deforming interfaces, and geometric algorithms for evolving and managing the resulting dynamic particle-mesh systems. The associated dynamic data structures are particularly troublesome on highly parallel computers. Even worse, there are many physicochemical effects which cannot be captured by continuum models. In addition, while one is interested in understanding the phenomena that give rise to the non-Newtonian rheological behavior of complex liquids, continuum approaches generally rely on the use of phenomenological constitutive relations to model this behavior.

In all these cases, the correct modeling of the phenomena of interest requires the use of "coarse-grained" mesoscopic approaches that mimic the behavior of atomistic systems on the length scales of interest. The goal is to reproduce the physics of fluid flow, primarily the conservation laws, while including the essential features of the underlying microscopic and mesoscopic physics. Two rather well known particle-based simulation techniques which have been developed with this goal in mind are Bird's DSMC method $[1,2]$ and dissipative particle dynamics (DPD) $[3,4]$.

Dissipative particle dynamics is an isothermal off-lattice technique which is essentially a molecular dynamics simulation in which each particle-which represents a mesoscopic element of the underlying molecular fluid-interacts with all the particles inside a sphere of radius $r_{0}$ through a conservative force, as well as dissipative forces which represent the coupling to a heat bath, and random forces which supply the energy lost by damping. In this coarse-grained description, the dominant interactions are the dissipative and random forces; the conservative forces are weak and of relatively 
long range. The soft nature of the DPD potentials allows for much longer time steps than in traditional MD simulations. Since DPD is an off-lattice technique, it is Galilean invariant.

It has been shown [5], using projection operator techniques, that DPD yields the correct macroscopic hydrodynamic equations, and kinetic theory was used to relate the transport coefficients in the hydrodynamic equations with the DPD model parameters [6]. The effect of finite time steps on the equilibrium state of the system has also been studied [7]. DPD has been used to study a rather large range of problems ranging from flow past complex objects [3], concentrated colloidal suspensions $[4,8,9]$, polymer suspensions $[10,11]$, and phase separation [12-14]. More recently, the DPD method has been generalized to include energy conservation [15-17], and the wave vector dependent transport properties of the DPD fluid have been calculated analytically using kinetic theory methods [18].

The direct simulation Monte Carlo algorithm $[1,2,19]$ is a stochastic, particle-based approach for solving the nonlinear time-dependent Boltzmann equation. In this approach, the system is partitioned into cells, with $\approx 50$ particles per cell. Each particle in the simulation is taken to effectively represent a large number of molecules in the physical system. For accurate calculations, the linear dimension of the cells must be less than the mean free path of the particles, and the general rule of thumb is that in regions with large gradients, the cell dimension should be approximately one-third the mean free path [2]. The algorithm consists of two steps, streaming and collision. In the streaming step, particles propagate freely for a time step $\tau_{d}$; collisions are then performed stochastically, with scattering rates and postcollision velocity distributions determined from dilute gas kinetic theory. Since the Boltzmann equation provides an accurate description of dilute gases arbitrarily far from equilibrium, even when there are large gradients in the hydrodynamic fields, this approach can be used to study phenomena outside the range of applicability of hydrodynamic equations. In fact, in 1989 the DSMC method was called [20,21] "the dominant predictive tool in rarefied gas dynamics for the past decade."

Comparisons of the results of the DSMC method and molecular dynamics simulations have shown that the DSMC method yields accurate results for shocks [22] and slip lengths [23], and many computational studies have shown that the DSMC results are in good agreement with solutions of the Navier-Stokes equation in the limit of very small Knudsen number [20]. For reviews of recent advances and applications of the DSMC method, see Refs. [24-26]. Some notable recent extensions of this approach include generalizations to model the Enskog equation for a hard-sphere fluid [27-29] and fluids with a van der Waals equation of state [30].

In this paper, we discuss an alternative particle-based simulation technique for modeling fluid dynamics [31-34], called stochastic rotation dynamics (SRD). It shares many features with the DSMC method and its variants, but differs in the nature of the collision rules. Like the DSMC method, the fluid is modeled by particles whose positions and velocities are continuous variables, and the system is coarse grained into the cells of a regular lattice with no restriction on the number of particles in a cell. The evolution of the system consists of two steps: streaming and collision. In the streaming step, the coordinate of each particle is incremented by its displacement during the time step. However, unlike the DSMC method, collisions are modeled by a simultaneous stochastic rotation of the relative velocities of every particle in each cell. The dynamics is explicitly constructed to conserve mass, momentum, and energy, and the collision process is the simplest consistent with these conservation laws. It has been shown that there is an $H$ theorem for the dynamics and that this procedure yields the correct hydrodynamic equations for an ideal gas [31].

The essential features of the algorithm are the following. Consider a set of $N$ point particles with (continuous) coordinates $\mathbf{r}_{i}(t)$ and velocities $\mathbf{v}_{i}(t)$. In the following, the mass of the particles is set equal to 1 . In the streaming step, all particles are propagated simultaneously a distance $\mathbf{v}_{i} \tau$, where $\tau$ is the value of the discretized time step. For the collision step, particles are sorted into cells, and they interact only with members of their own cell. Typically, the simplest cell construction consisting of a hypercubic grid with mesh size $a$ is used. The collision step consists of an independent random rotation of the relative velocities, $\mathbf{v}_{i}-\mathbf{u}$ of the particles in each cell, where the macroscopic velocity $\mathbf{u}(\boldsymbol{\xi}, t)$ is the mean velocity of the particles in the cell with coordinate $\boldsymbol{\xi}$. The local temperature $T(\xi, t)$ is defined via the mean square deviation of the particle velocities from the mean velocity in a cell. All particles in a cell are subject to the same rotation, but the rotation angles of different cells are statistically independent. There is a great deal of freedom in how the rotation step is implemented $[31,33,35]$, since, by construction, the local momentum and kinetic energy are invariant. The dynamics is therefore summarized by

$$
\begin{gathered}
\mathbf{r}_{i}(t+\tau)=\mathbf{r}_{i}(t)+\tau \mathbf{v}_{i}(t), \\
\mathbf{v}_{i}(t+\tau)=\mathbf{u}\left[\xi_{i}(t+\tau)\right]+\boldsymbol{\omega}\left[\boldsymbol{\xi}_{i}(t+\tau)\right] \cdot\left\{\mathbf{v}_{i}(t)-\mathbf{u}\left[\xi_{i}(t+\tau)\right]\right\},
\end{gathered}
$$

where $\boldsymbol{\omega}\left(\boldsymbol{\xi}_{i}\right)$ denotes a stochastic rotation matrix, and $\boldsymbol{\xi}_{i}$ is the coordinate of the cell occupied by particle $i$ at the time of the collision. $\mathbf{u}(\boldsymbol{\xi}) \equiv(1 / M) \Sigma_{k \in \xi} \mathbf{v}_{k}$ is the mean velocity of the particles in cell $\boldsymbol{\xi}$. In two dimensions, $\boldsymbol{\omega}$ is typically taken to be a rotation by an angle $\pm \alpha$, with probability $1 / 2$. However, any stochastic rotation matrix consistent with detailed balance can be used. Several choices in three dimensions are discussed in Ref. [35].

\section{A. Galilean invariance and molecular chaos}

In order to perform the collision operation, the system is coarse grained into the cells of a regular lattice. This breaks Galilean invariance, since the collision environment of any given particle depends on the value of a superimposed constant velocity field. Consider the difference between a stationary medium and one that moves with a constant velocity U. Unless the displacement of the moving system in one time step is exactly commensurate with the cell structure, a particle in the moving system will find that its position relative to the cell boundaries is different than in the stationary 


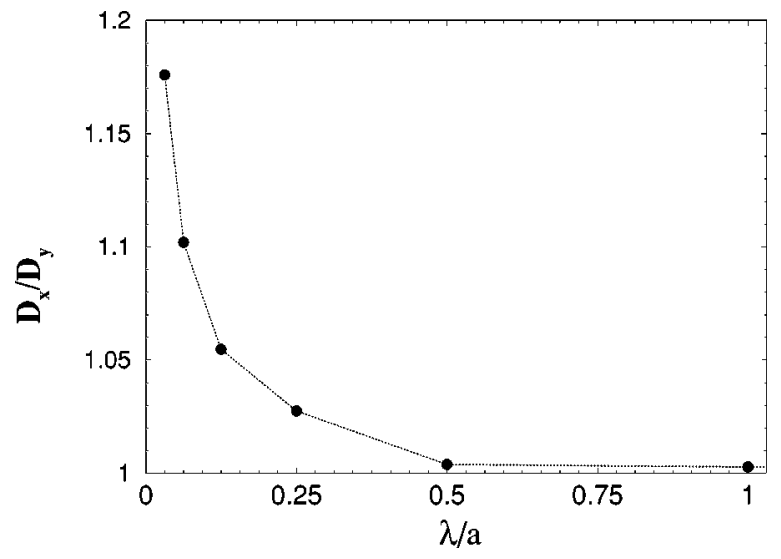

FIG. 1. Test of Galilean invariance. The ratio of the selfdiffusion coefficients $D_{x}$ and $D_{y}$ in $x$ and $y$ directions plotted as a function of the ratio of the mean free path $\lambda$ to the cell size $a$. A homogenous flow in $x$ direction, $\mathbf{u}=(0.2,0)$, is imposed, and no random shift has been applied. Parameters: $k_{B} T=0.0625, M=35$, and $L=32$.

frame. Because of this, the particles participating in the collision process will be different in the stationary and moving frames, thus breaking Galilean invariance. This breakdown of Galilean invariance is negligible if the mean free path $\lambda$ $=\tau \sqrt{k_{B} T}$ of the particles is large compared to the cell size $a$. In this case, molecular chaos is a valid assumption because most collisions involve particles that have just arrived from different cells, and are therefore not correlated. After a collision, particles immediately leave to other cells, and hence decorrelate quickly. There are no correlations which can be affected by a homogeneous flow field. The breakdown of the Galilean invariance can, however, become significant for small $\lambda / a$. In the limit of small mean free path, essentially the same set of particles "collide" several times before some of the participating particles leave the cell or other particles enter. The particles are therefore correlated prior to the collision step, and the degree of correlation depends on the value of the imposed flow field. The transport coefficients depend on the value of $\mathbf{U}$, and Galilean invariance is broken. This behavior is confirmed by simulations. For example, simulation results for the ratio of the self-diffusion constants in $x$ and $y$ directions, $R=D_{x} / D_{y}$, measured in a homogeneous flow field as a function of $\lambda / a$ are presented in Fig. 1 . While there are significant deviations from $R=1$ for small $\lambda / a$, for $\lambda>a / 2, R$ deviates from 1 by less than $0.3 \%$.

Consider now what happens if, before the collision step, all particles are shifted by the same uniformly distributed random translation vector with components in the interval $[-a / 2, a / 2]$ before the collision step. The shift randomizes the position of particles in the cell, and any given particle has an equal probability to be found at any position in a cell. This is true in both stationary and moving frames. There is therefore an equal probability that the outcome of the collision process is the same in both frames, so that Galilean invariance is exactly restored. This makes it possible to perform simulations at arbitrary Mach number, even for low temperature, at little additional computational cost. In our implementation of this procedure all particles are shifted by the same random vector with components in the interval $[-a / 2, a / 2]$ before the collision step. Particles are then shifted back to their original positions after the collision. If we denote the cell coordinate of the shifted particle $i$ by $\boldsymbol{\xi}_{i}^{s}$, the collision step is described by

$$
\mathbf{v}_{i}(t+\tau)=\mathbf{u}\left[\boldsymbol{\xi}_{i}^{S}(t+\tau)\right]+\boldsymbol{\omega}\left[\boldsymbol{\xi}_{i}^{S}(t+\tau)\right] \cdot\left\{\mathbf{v}_{i}(t)-\mathbf{u}\left[\boldsymbol{\xi}_{i}^{S}(t+\tau)\right]\right\}
$$

instead of Eq. (2).

The random shifts also rectify an unphysical property of the multiparticle interaction provided by the rotation. The partitioning of the particles into cells means that there is the possibility that there is no interaction between two particles even if they are infinitesimally close, but separated by a cell boundary, while other particles in the same cell interact nonlocally up to a distance $a \sqrt{2}$. On average, the interaction between two particles is a smooth function of the distance (and the orientation of the connecting vector) between two particles if random shifts are applied.

The random shift procedure is also essential for generalizations of this model to nonideal gases [36] where a phase boundary between fluid and gas phases can occur. Without the modification, a homogeneous flow would lead to a deformation of a spherical droplet at small mean free path.

Finally, note that the random shift procedure does not alter the anisotropy caused by the underlying cubic grid. In dimension $d=2$, the anisotropy can be reduced by using a hexagonal cell structure. More generally, additional random rotations of the grid should be applied in order to achieve full isotropy. We will assume that the anisotropy is small, at least at moderate and large mean free path, and will not consider it here.

\section{B. Detailed balance and the $\boldsymbol{H}$ theorem}

It was shown in Ref. [31] that there is a Boltzmann $H$ theorem for the SRD algorithm if (a) the stochastic collision rules satisfy semidetailed balance and (b) Boltzmann's assumption of molecular chaos is valid. The assumption of molecular chaos is required in order to be able to write the full $N$-particle probability distribution function as the product of identical single-particle distributions, and semidetailed balance is required to guarantee that the $H$ functional

$$
H_{B}(t)=\int d \mathbf{v} d \mathbf{r} f(\mathbf{v}, \mathbf{r}, t) \ln f(\mathbf{v}, \mathbf{r}, t),
$$

where $f(\mathbf{v}, \mathbf{r}, t)$ is the reduced single-particle distribution function, decreases with time and attains a minimum for the equilibrium Maxwell distribution,

$$
f=f_{e q} \equiv \frac{N}{V}\left(\frac{1}{2 \pi k_{B} T}\right)^{d / 2} \exp \left[(\mathbf{v}-\mathbf{u})^{2} / 2 k_{B} T\right]
$$

The $H$ theorem guarantees that the dynamical system relaxes to the correct Gibbs equilibrium state. Furthermore, it ensures that there are no numerical instabilities associated with 
a nonmonotonic decay of the $H$ functional, as are often found in interacting lattice-gas and lattice-Boltzmann models $[37,38]$.

As discussed in Sec. I A, the assumption of molecular chaos is not valid for the SRD algorithm because of the cell structure introduced to define the collision environment. Although the Stosszahlansatz is not a bad approximation for large mean free paths, $\lambda>a$, it breaks down completely when $\lambda$ is significantly smaller then the cell size, since correlations build up over several collisions. It follows that an $H$ theorem of the type discussed in the last paragraph is not strictly valid. Nevertheless, there is a more general type of $H$ theorem which the SRD algorithm does satisfy. In general, any Markov chain or process with an equilibrium distribution will have an $H$ theorem associated with it, in the sense that there is a whole class of Lyapunov functions that decrease monotonically with time. Any convex function of the $N$-particle distribution achieves this. In particular, Hénon has shown quite generally [39] that the assumption of semidetailed balance in such a process is sufficient to prove an $H$ theorem of this type. More generally, the $H$ theorem follows directly from the master equation for the $\mathrm{N}$-particle distribution function if the Markov process satisfies a detailed balance condition $[38,40]$. In the remainder of this section we show that the collision dynamics of the SRD algorithm with random cell shifts satisfies a detailed balance condition and discuss the resulting $H$ theorem.

Malevanets and Kapral [31] have shown that the original SRD algorithm conserves the elementary measure $d \Gamma$ $=\Pi d \mathbf{r}_{i} d \mathbf{v}_{i}$. For the streaming step, Eq. (1), it is clear that the Jacobian is 1 . The collision step is a rotation of the relative momenta. Semidetailed balance and the fact that the choice of rotation does not depend on the relative momenta ensure that the Jacobian of this operation is also equal to 1, so that the phase space measure is invariant. Note that this remains true even if the random shift operation discussed in the previous section is implemented. The evolution in phase space is Markovian, and the Liouville equation for the evolution of the $N$-particle probability distribution in the original $\mathrm{SRD}$ algorithm is [31]

$$
P\left(\mathbf{V}^{(N)}, \mathbf{R}^{(N)}+\tau \mathbf{V}^{(N)}, t+\tau\right)=C_{\mathbf{b}^{\circ}} P\left(\mathbf{V}^{(N)}, \mathbf{R}^{(N)}, t\right),
$$

where the action of the collision operator $C_{\mathbf{b}}$ is given by

$$
\begin{aligned}
C_{\mathbf{b}} \circ & P\left(\mathbf{V}^{(N)}, \mathbf{R}^{(N)}, t\right) \\
= & \frac{1}{2^{Q}} \sum_{\{\omega\}} \int d \breve{\mathbf{R}}^{(N)} P\left(\breve{\mathbf{V}}^{(N)}, \mathbf{R}^{(N)}, t\right) \\
& \times \prod_{i=1}^{N} \delta\left(\mathbf{v}_{i}-\mathbf{u}_{\xi_{i}^{b}}-\boldsymbol{\omega}\left(\check{\boldsymbol{\xi}}_{i}^{\mathbf{b}}\right) \cdot\left[\breve{\mathbf{v}}_{i}-\mathbf{u}_{\xi_{i}^{\mathbf{b}}}\right]\right),
\end{aligned}
$$

where $Q$ is the number of simulation cells, $\mathbf{V}^{(N)}$ $=\left(\mathbf{v}_{1}, \ldots, \mathbf{v}_{N}\right), \mathbf{R}^{(N)}=\left(\mathbf{r}_{1}, \ldots, \mathbf{r}_{N}\right)$, and $\breve{\mathbf{V}}^{(N)}$ denotes the velocities before the rotation. $\boldsymbol{\xi}^{\boldsymbol{b}}$ is the cell coordinate (see Sec. II A), and $\mathbf{b}$ indexes a particular choice of origin for the grid (e.g., the position of a particular cell center), which is fixed but arbitrary in the original algorithm.
In the following discussion, we consider random grid shifts, while keeping the particle positions fixed. This is equivalent to the procedure described in the preceding section. The functional form of Eq. (6) is independent of the choice of grid origin; the center of mass velocities of a cell, and the sets of collision partners will, of course, depend on the particular choice of $\mathbf{b}$. The grid shift before the collision step entails a displacement of the grid by a random translation vector with components uniformly distributed in the interval $[-a / 2, a / 2]$. Because the size of the random translation does not depend on either the particle positions or velocities, and is uncorrelated in time, the Liouville operator for the system with random shift is simply a superposition of the operator for a fixed grid. Equation (6) therefore becomes

$$
\begin{aligned}
& P\left(\mathbf{V}^{(N)}, \mathbf{R}^{(N)}+\tau \mathbf{V}^{(N)}, t+\tau\right) \\
& \quad=\frac{1}{a^{d}} \int_{-a / 2}^{a / 2} \ldots \int_{a / 2}^{a / 2} d b_{1} \cdots d b_{d} C_{\mathbf{b}^{\circ}} P\left(\mathbf{V}^{(N)}, \mathbf{R}^{(N)}, t\right) .
\end{aligned}
$$

For any fixed grid, the Maxwell distribution is a fix point of the Liouville equation [31], and because of Eq. (8) it remains a fix point with random shifts. Assuming molecular chaos, this means that the $H$ theorem proven in Ref. [31] remains valid when random grid shifts are performed. This is supported by simulations, which showed convergence to the Maxwell distribution, both with and without random cell shifts. There were no instabilities, and the final state is always spatially homogeneous; for large mean free path, both the dynamics and transport coefficients are not changed.

In general, however, molecular chaos is not a valid assumption, and there is no $H$ theorem for functional (4). Nevertheless, there is a more general type of $H$ theorem describing the relaxation of the $\mathrm{N}$-particle distribution function. As discussed above, the SRD collision rules must satisfy a detailed balance condition in order for this to be true. In two dimension, where the stochastic rotation matrix is a rotation by an angle $\pm \alpha$, with probability $1 / 2$, detailed balance is clearly satisfied. More generally, detailed balance requires that the inverse rotation can occur with equal probability. Consider a system of $N$ particles, with a fixed grid consisting of $Q$ cells. The microscopic state of this system, $\left\{\mathbf{r}_{i}, \mathbf{v}_{i}\right\}$, will be denoted by $A$. Let $B$ be the state after one streaming and collision step. The transition probability from $A$ to $B, w(A$ $\rightarrow B$ ), is proportional to $1 / 2^{Q}$, because we have two possibilities for the rotation in every cell. Now invert time, $\tau \rightarrow-\tau$, and perform another collision and streaming step. The probability of obtaining state $A, w(B \rightarrow A)$, is exactly the same as $w(A \rightarrow B)$, so that detailed balance is realized.

Consider now the effect of the grid shift. Starting from configuration $A$, the streaming step is performed and the grid is shifted by a particular vector $\mathbf{b}$. The velocities are rotated by the angle $\alpha$, and a new state $B$ is obtained. The grid is now shifted back to its original position. Because of the shift, there are many more possibilities for obtaining configuration $B$ than without shifting. However, after time reversal, the old state $A$ can be obtained again with the same probability, since one needs again a random shift by the same vector $\mathbf{b}$ and a rotation by angle $-\alpha$ to attain this state. The probability of 
choosing the same $\mathbf{b}$ for the inverse operation is the same as for the forward time step time, since the selection of $\mathbf{b}$ is independent of time and state of the system. Detailed balance is therefore not affected by the shift, so the conditions for an $H$ theorem are fulfilled.

In principle, any convex function of the $N$-particle probability distribution can be used to define $H$, but the standard choice,

$$
H(t)=\int d \Gamma P \ln P
$$

where the $N$-particle distribution $P=P\left(\mathbf{V}^{(N)}, \mathbf{X}^{(N)}, t\right)$, and $d \Gamma=d \mathbf{V}^{(N)} d \mathbf{X}^{(N)}$ ensures that the Maxwell distribution minimizes $H$. To see this, note that since $P \ln P$ is a convex function of $P$,

$$
\begin{aligned}
H(t)-H_{e q}= & \int d \Gamma\left(P \ln P-P_{e q} \ln P_{e q}\right) \geqslant \int d \Gamma\left(\ln P_{e q}+1\right) \\
& \times\left(P-P_{e q}\right)
\end{aligned}
$$

where, for the moment, $P_{e q}$ is arbitrary. However, the Maxwell distribution

$$
P_{e q} \sim \exp \left(-\sum_{i}^{N}\left(\mathbf{v}_{i}-\mathbf{u}\right)^{2} / 2 k_{B} T\right)
$$

provides a lower bound for the $H$-functional, Eq. (9), because conservation of mass, momentum, and energy guarantees that

$$
\int d \Gamma P \ln P_{e q}=\int d \Gamma P_{e q} \ln P_{e q}=H_{e q}
$$

and

$$
\int d \Gamma P=\int d \Gamma P_{e q} .
$$

The right hand side of Eq. (10) is therefore zero for $P_{e q}$ given by Eq. (11), proving the $H$ theorem.

\section{Outline}

The remainder of the paper is organized as follows. In Sec. II, we introduce our choice of coarse-grained hydrodynamic variables and derive the equations of motion for the correlation functions using a discrete-time projection operator formalism similar to that utilized by Dufty and Ernst [41] in their derivation of the Green-Kubo relations for lattice-gas cellular automata. The shifting procedure discussed above is incorporated into the formalism, and it is shown that while it does not change the reversible Euler terms in the equations of motion, it does lead to new contributions to the GreenKubo relations for the transport coefficients. In Sec. III, numerical results for the rate of decay to thermal equilibrium and the shear viscosity are presented. In particular, the viscosity is determined from both the rate of decay of vorticity correlations and the linear response of the system to an imposed $k$-dependent force. It is shown that there are $k$-dependent anomalies in the viscosity and that the measured values of the viscosity depend on the direction and magnitude of an external homogeneous flow field if there are no random cell shifts before collisions. It is then shown that the shifting procedure removes the anomalies.

In the following paper (referred to hereafter as Part 2), calculations of the leading terms of the stress correlation functions are presented, which take explicit account of the cell structure. It is shown that finite cell size effects can persist even in the large mean free path regime and that they are the cause of previous discrepancies between analytic and simulation results in $d=2$ for $\alpha \approx 90^{\circ}$. Explicit results for all the transport coefficients in $d=2$ are derived and shown to be in excellent agreement with simulation results for all $\lambda / a$. Finally, long-time tails in the velocity, shear stress, and heatflux autocorrelations are measured and compared with the predictions of mode-coupling theory.

\section{HYDRODYNAMICS}

\section{A. Definition of hydrodynamic variables}

The density, momentum, and energy density operators are

$$
A_{\beta}(\mathbf{r})=\sum_{i=1}^{N} a_{\beta, i} \delta\left(\mathbf{r}-\mathbf{r}_{i}\right),
$$

where $a_{1, i}=1$ for the density, $\left\{a_{\beta, i}\right\}=\left\{v_{i(\beta-1)}\right\}$, with $\beta$ $=2, \ldots, d+1$, are the components of the particle momenta, and $a_{d+2, i}=v_{i}^{2} / 2$ is the kinetic energy of particle $i$. Cell operators $\left\{A_{\beta}^{c}(\xi)\right\}$ can be defined as integrals over the cell volume of the density operators:

$$
\begin{aligned}
A_{\beta}^{c}(\boldsymbol{\xi}) & =\sum_{i=1}^{N} \int d \mathbf{r} a_{\beta, i} \delta\left(\mathbf{r}-\mathbf{r}_{i}\right) \prod_{\gamma=1}^{d} \Theta\left(\frac{a}{2}-\left|\xi_{\gamma}-r_{\gamma}\right|\right) \\
& =\sum_{i=1}^{N} a_{\beta, i} \prod_{\gamma=1}^{d} \Theta\left(\frac{a}{2}-\left|\xi_{\gamma}-r_{i \gamma}\right|\right)
\end{aligned}
$$

with the discrete cell coordinates $\boldsymbol{\xi}=a \mathbf{m}$, with $m_{\beta}$ $=1, \ldots, L$, for each spatial component.

The Fourier transform of the densities is

$$
A_{\beta}(\mathbf{k})=\int_{V} A_{\beta}(\mathbf{r}) e^{i \mathbf{k} \cdot \mathbf{r}} d \mathbf{r}
$$

where $V$ is the volume of the system. The inverse transform is

$$
A_{\beta}(\mathbf{r})=\frac{1}{V} \sum_{\mathbf{k}} A_{\beta}(\mathbf{k}) e^{-i \mathbf{k} \cdot \mathbf{r}} .
$$

For a $d$-dimensional system of volume $V=(a L)^{d}$ with periodic boundary conditions, $\mathbf{k}=2 \pi \mathbf{n} /(a L)$, where $n_{\beta}=0$, $\pm 1, \ldots$ for all spatial components.

The Fourier transforms of the cell variables are

$$
A_{\beta}^{c}(\mathbf{q})=\sum_{\xi} A_{\beta}^{c}(\boldsymbol{\xi}) e^{i \mathbf{q} \cdot \xi}=\sum_{j} a_{\beta, j} e^{i \mathbf{q} \cdot \xi_{j}}
$$


where $\boldsymbol{\xi}_{j}$ is the coordinate of the cell occupied by particle $j$. The inverse transforms are

$$
A_{\beta}^{c}(\boldsymbol{\xi})=\frac{1}{L^{d}} \sum_{\mathbf{q}} A_{\beta}^{c}(\mathbf{q}) e^{-i \mathbf{q} \cdot \boldsymbol{\xi}},
$$

where the wave vectors $\mathbf{q}=2 \pi \mathbf{n} /(a L)$, where $n_{\beta}=0$, $\pm 1, \ldots, \pm(L-1), L$ for the spatial components. The Fourier transform (16) can also be applied to cell variables if we define densities

$$
A_{\beta}(\mathbf{r})=\left[A_{\beta}^{c}(\xi) / a^{d}\right] \prod_{\gamma=1}^{d} \Theta\left(\frac{a}{2}-\left|\xi_{\gamma}-r_{\gamma}\right|\right) .
$$

In this case, one has

$$
A_{\beta}(\mathbf{k})=A_{\beta}^{c}(\mathbf{k}) f(a \mathbf{k}),
$$

where

$$
f(a \mathbf{k})=\prod_{\gamma=1}^{d} \frac{2 \sin \left(a k_{\gamma} / 2\right)}{a k_{\gamma}}
$$

is the form factor of a cell. The first term on the right hand side of Eq. (21) is the cell transform (18).

There is a certain amount of freedom when identifying coarse-grained densities in this model. For the momentum and energy densities, the cell densities are the obvious choices, since Eq. (2) has the collision invariants

$$
\sum_{j} e^{i \mathbf{q} \cdot \xi_{j}(t+\tau)}\left[a_{\beta, j}(t+\tau)-a_{\beta, j}(t)\right]=0 .
$$

It would therefore be natural to also use the cell particle density. If this is done, however, one finds that there is a small anomalous dissipative term (of order wave vector squared) in the hydrodynamic equation for the density. The reason for this is that for this choice of variables, the cancellation of terms in the corresponding Green-Kubo relation for the particle density is incomplete. One way around this problem is to use the particle density defined in Eq. (14) and the corresponding Fourier transform (16). For the momentum and energy densities, the corresponding Fourier transforms are given by Eq. (21).

The choice of coarse-grained hydrodynamic variables is reflected in the detailed form of the Green-Kubo relations for the transport coefficients. Note, however, that the physics of the problem, i.e., the hydrodynamic modes, are not affected by the particular choice of the density operator. Any additional contributions to the continuity equation will be canceled by other spurious terms when calculating the hydrodynamic modes.

\section{B. Equations of motion for the correlation functions}

The projection operator technique introduced by Zwanzig [42-44] provides a convenient formalism for deriving the linearized hydrodynamic equations from the microscopic equations of motion (1) and (3). With the help of this technique, explicit expressions for both the reversible (Euler) as well as dissipative terms of the long-time large-length-scale hydrodynamics equations for the coarse-grained hydrodynamic variables can be derived. In addition, Green-Kubo relations are obtained which enable explicit calculations of the transport coefficients of the fluid.

In the following, we summarize results for the equations of motion for the equilibrium correlation functions of the coarse-grained conserved variables. Since the correlation functions define the linear response of the average value of the conserved quantities, the same approach could also be used to determine the equations of motion for the average values of the conserved variables. The equilibrium correlation functions for the conserved variables (denoted collectively by $\left.A_{\beta}\right)$, are defined by $\left\langle\delta A_{\beta}(\mathbf{r}, t) \delta A_{\gamma}\left(\mathbf{r}^{\prime}, t\right)\right\rangle$, where $\langle\delta A\rangle=A-\langle A\rangle$, and the brackets denote an average over the equilibrium distribution. In a stationary, translationally invariant system of the type considered here, the correlation function depends only on the differences $\mathbf{r}-\mathbf{r}^{\prime}$ and $t-t^{\prime}$, and the Fourier transform of the correlation function, which we shall denote by $G_{\beta \gamma}(\mathbf{k}, t) \equiv\left\langle A_{\beta}(\mathbf{k}, 0) \mid A_{\gamma}(\mathbf{k}, t)\right\rangle$, is

$$
G_{\beta \gamma}(\mathbf{k}, t)=V^{-1}\left\langle\delta A_{\beta}^{*}(\mathbf{k}, 0) \delta A_{\gamma}(\mathbf{k}, t)\right\rangle,
$$

where the asterisk denotes complex conjugation. To simplify notation, we omit the wave vector dependence in the correlation function in the remainder of this section.

Including the shift operation, the dynamics described by Eqs. (1) and (3) consists of consecutive streaming and collision operations. Since particles are neither created or destroyed, the number of particles is trivially conserved. The collision invariants for the momentum and energy density are

$$
\sum_{j} e^{i \mathbf{k} \cdot \xi_{j}^{s}(t+\tau)}\left[a_{\beta, j}(t+\tau)-a_{\beta, j}(t)\right]=0,
$$

where $\boldsymbol{\xi}_{j}^{s}$ is the coordinate of the cell occupied by particle $j$ in the shifted system. These conservation laws can be written as

$$
\Delta_{t} A_{\beta}(t)+i \mathbf{k} \cdot \mathbf{D}_{\beta}(t)=0,
$$

where $\Delta_{t} A(t)=[A(t+\tau)-A(t)] / \tau$ and the flux $\mathbf{D}_{\beta}$ is given by

$$
\mathbf{D}_{1}(t)=\frac{1}{\tau} \sum_{j} \frac{\Delta \mathbf{r}_{j}(t) e^{i \mathbf{k} \cdot \mathbf{r}_{j}(t)}}{i \mathbf{k} \cdot \Delta \mathbf{r}_{j}(t)}\left[1-e^{i \mathbf{k} \cdot \Delta \mathbf{r}_{j}(t)}\right]
$$

where $\Delta \mathbf{r}_{j}(t)=\mathbf{r}_{j}(t+\tau)-\mathbf{r}_{j}(t)$, for the particle density, and

$$
\begin{aligned}
\mathbf{D}_{\beta}(t)= & \frac{1}{\tau} \sum_{j}\left\{\frac{a_{\beta, j}(t) \Delta \boldsymbol{\xi}_{j}(t) e^{i \mathbf{k} \cdot \xi_{j}(t)}}{i \mathbf{k} \cdot \Delta \boldsymbol{\xi}_{j}(t)}\left[1-e^{i \mathbf{k} \cdot \Delta \xi_{j}(t)}\right]\right. \\
& +\frac{\Delta a_{\beta, j}(t) \Delta \boldsymbol{\xi}_{j}^{s}(t) e^{i \mathbf{k} \cdot \xi_{j}(t)}}{i \mathbf{k} \cdot \Delta \boldsymbol{\xi}_{j}^{S}(t)}\left[e^{-i \mathbf{k} \cdot \Delta \xi_{j}^{(}(t)}\right. \\
& \left.-1] e^{i \mathbf{k} \cdot \Delta \boldsymbol{\xi}_{j}(t)}\right\} f(a \mathbf{k})
\end{aligned}
$$

where $\quad \Delta \boldsymbol{\xi}_{j}(t)=\boldsymbol{\xi}_{j}(t+\tau)-\boldsymbol{\xi}_{j}(t), \quad \Delta \boldsymbol{\xi}_{j}^{s}(t)=\boldsymbol{\xi}_{j}(t+\tau)-\boldsymbol{\xi}_{j}^{s}(t$ $+\tau)$, and $\Delta a_{\beta, j}(t)=a_{\beta, j}(t+\tau)-a_{\beta, j}(t)$, for the momentum 
and energy densities. The conservation laws (26) imply the following equations for correlation functions of the conserved variables and their fluxes:

$$
\begin{gathered}
\Delta_{t}\langle A \mid A(t)\rangle+i \mathbf{k} \cdot\langle A \mid \mathbf{D}(t)\rangle=0, \\
\Delta_{t}\left\langle D_{i} \mid A(t)\right\rangle+i \mathbf{k} \cdot\left\langle D_{i} \mid \mathbf{D}(t)\right\rangle=0,
\end{gathered}
$$

where $A=A(0)$ and, similarly, $D_{i}=D_{i}(0)$. Finally, stationarity implies the relation

$$
\langle A \mid \mathbf{D}(t)\rangle=\langle\mathbf{D} \mid A(t+\tau)\rangle,
$$

or, using Eq. (29),

$$
\Delta_{t}\langle A \mid A(t)\rangle+i \mathbf{k} \cdot\langle\mathbf{D} \mid A(t+\tau)\rangle=0 .
$$

Introducing the discrete Laplace transforms

$$
\begin{aligned}
& |\widetilde{A}(s)\rangle=\sum_{n=0}^{\infty} e^{-s \tau n}|A((n+1) \tau)\rangle, \\
& |\widetilde{\mathbf{D}}(s)\rangle=\sum_{n=0}^{\infty} e^{-s \tau n}|\mathbf{D}(n \tau)\rangle,
\end{aligned}
$$

Eqs. (29), (30), and (32) can be written as

$$
\begin{gathered}
\left(1-e^{-s \tau}\right)\langle A \mid \widetilde{A}(s)\rangle+i \tau \mathbf{k} \cdot\langle A \mid \widetilde{\mathbf{D}}(s)\rangle=\langle A \mid A\rangle, \\
\left(1-e^{-s \tau}\right)\left\langle D_{i} \mid \widetilde{A}(s)\right\rangle+i \tau \mathbf{k} \cdot\left\langle D_{i} \mid \widetilde{\mathbf{D}}(s)\right\rangle=\left\langle D_{i} \mid A\right\rangle, \\
\left(1-e^{-s \tau}\right)\langle A \mid \widetilde{A}(s)\rangle+i \tau \mathbf{k} \cdot\langle\mathbf{D} \mid \widetilde{A}(s)\rangle=\langle A \mid A\rangle .
\end{gathered}
$$

Note that with definitions (33), the Laplace transform of Eq. (31) is

$$
\langle A \mid \widetilde{\mathbf{D}}(s)\rangle=\langle\mathbf{D} \mid \widetilde{A}(s)\rangle .
$$

In order to determine the linearized hydrodynamics equations corresponding to the dynamics described by Eqs. (34)(36), we follow Ref. [41] and introduce a formal linear hydrodynamics for $\langle A \mid \widetilde{A}(s)\rangle$ by the equation

$$
\left[\left(1-e^{-s \tau}\right)+\tau L(\mathbf{k}, s)\right]\langle A \mid \widetilde{A}(s)\rangle=\langle A \mid A\rangle .
$$

The linear hydrodynamic equations describe the long-time large-length-scale dynamics of the system, and they are valid in the limits of small $k$ and $s$. To evaluate $L$ is this limit, first note that a comparison of Eqs. (36) and (38) shows that

$$
L(\mathbf{k}, s)\langle A \mid \widetilde{A}(s)\rangle=i \mathbf{k} \cdot\langle\mathbf{D} \mid \widetilde{A}(s)\rangle .
$$

Multiplying this result by $\left(1-e^{-s \tau}\right)$ and using Eqs. (34) and (35), we have

$$
L[\langle A \mid A\rangle-i \tau \mathbf{k} \cdot\langle A \mid \widetilde{\mathbf{D}}(s)\rangle]=i k_{i}\left[\left\langle D_{i} \mid A\right\rangle-i \tau k_{j}\left\langle D_{i} \mid \widetilde{D}_{j}(s)\right\rangle\right],
$$

which shows that $L$ is of order of $k$. Solving Eq. (40) for $L$ to order $k^{2}$ yields

$$
L=i k_{i}\left\langle D_{i} \mid A\right\rangle\langle A \mid A\rangle_{0}^{-1}+\tau k^{2}\langle I \mid \widetilde{I}(s)\rangle\langle A \mid A\rangle_{0}^{-1},
$$

where

$$
k^{2}\langle I \mid \widetilde{I}(s)\rangle=k_{i} k_{j}\left[\left\langle D_{i} \mid \widetilde{D}_{j}(s)\right\rangle-\left\langle D_{i} \mid A\right\rangle\langle A \mid A\rangle_{0}^{-1}\left\langle A \mid \widetilde{D}_{j}(s)\right\rangle\right] .
$$

In Eq. (41), $\langle A \mid A\rangle_{0}$ is the $k \rightarrow 0$ limit of the susceptibility matrix. Since the form factor $f(a \mathbf{k}) \simeq 1-a^{2} k^{2} / 24$ for small $k$, only the zero wave vector limit of the susceptibility matrix contributes to $L$ to this order in $k$. The reduced flux

$$
I_{\beta}(\hat{\mathbf{k}})=\hat{\mathbf{k}} \cdot\left[\mathbf{D}_{\beta}-\left\langle\mathbf{D}_{\beta} \mid A_{\gamma}\right\rangle\left(\langle A \mid A\rangle_{0}\right)_{\gamma \varepsilon}^{-1} A_{\varepsilon}\right]
$$

is the component of $\hat{\mathbf{k}} \cdot \mathbf{D}_{\beta}$ that is orthogonal to the conserved variables.

The first term on the right side of Eq. (41) also contains contributions of the order of $k^{2}$. In particular, Eq. (26) implies

$$
\tau\left\langle\mathbf{k} \cdot \mathbf{D}_{\beta} \mid \mathbf{k} \cdot \mathbf{D}_{\gamma}\right\rangle=i\left\langle\mathbf{k} \cdot \mathbf{D}_{\beta} \mid A_{\gamma}(\tau)\right\rangle-i\left\langle\mathbf{k} \cdot \mathbf{D}_{\beta} \mid A_{\gamma}\right\rangle,
$$

so that stationarity, Eq. (31), yields

$$
\begin{gathered}
\tau\left\langle\mathbf{k} \cdot \mathbf{D}_{\beta} \mid \mathbf{k} \cdot \mathbf{D}_{\gamma}\right\rangle=i\left\langle\mathbf{k} \cdot \mathbf{D}_{\beta} \mid A_{\gamma}\right\rangle^{*}-i\left\langle\mathbf{k} \cdot \mathbf{D}_{\beta} \mid A_{\gamma}\right\rangle \\
=2 \operatorname{Im}\left[\left\langle\mathbf{k} \cdot \mathbf{D}_{\beta} \mid A_{\gamma}\right\rangle\right] .
\end{gathered}
$$

It follows that

$$
i k_{i}\left\langle D_{i} \mid A\right\rangle=i k \omega-\frac{1}{2} \tau k_{i} k_{j}\left\langle D_{i}^{0} \mid D_{j}^{0}\right\rangle+O\left(k^{3}\right),
$$

where

$$
\left.\omega \equiv \hat{\mathbf{k}} \cdot\left\langle\mathbf{D}_{i}^{0} \mid A\right\rangle\right|_{k=0},
$$

and $\mathbf{D}^{0}$ is the small $k$ limit of $\mathbf{D}$. The frequency matrix $\Omega$ $\left.\equiv \omega\langle A \mid A\rangle^{-1}\right|_{k=0}$ contains the reversible (Euler) terms of the hydrodynamic equations.

In particular, for the particle density,

$$
\mathbf{D}_{1}^{0}=-\frac{1}{\tau} \sum_{j} \Delta \mathbf{r}_{j} e^{i \mathbf{k} \cdot \mathbf{r}_{j}},
$$

while for the momentum and energy densities,

$$
\mathbf{D}_{\beta}^{0}=-\frac{1}{\tau} \sum_{j}\left\{a_{\beta, j} \Delta \boldsymbol{\xi}_{j}+\Delta a_{\beta, j} \Delta \boldsymbol{\xi}_{j}^{\xi}\right\} e^{i \mathbf{k} \cdot \xi_{j}}
$$

The hydrodynamic poles of Eq. (38) are proportional to $k$ as $k \rightarrow 0$. It follows that to order $k^{2}$, these poles are given by the solution of the quadratic equation

$$
\tau s^{2}-2 s-2\left(i k \Omega+\Gamma k^{2}\right)=0,
$$

where

$$
\Gamma=\tau\left[\langle I \mid \widetilde{I}(s)\rangle-\frac{1}{2} \hat{k}_{i} \hat{k}_{j}\left\langle D_{i}^{0} \mid D_{j}^{0}\right\rangle\right]\langle A \mid A\rangle_{0}^{-1},
$$

so that 


$$
\begin{aligned}
{\left[\left(1-e^{-s \tau}\right)+\tau L(\mathbf{k}, s)\right] \rightarrow } & -\frac{\tau}{2}\left(s+i k \Omega+\Lambda k^{2}\right)(s \tau-2 \\
& \left.-i \tau k \Omega-\tau \Lambda k^{2}\right)
\end{aligned}
$$

where $\Lambda$ is given by the small $s$ limit of

$$
\Lambda(s)=\Gamma+\frac{\tau}{2} \Omega^{2}=\tau\left[\langle I \mid \widetilde{I}(s)\rangle-\frac{1}{2}\langle I \mid I\rangle\right]\langle A \mid A\rangle_{0}^{-1} .
$$

Combining these results, the linearized hydrodynamic equations of the system are therefore

$$
\left[s+i k \Omega+k^{2} \Lambda\right]\langle A \mid \widetilde{A}(s)\rangle=\frac{1}{\tau}\langle A \mid A\rangle R(k),
$$

where $R(k)=\left[1+\tau\left(i k \Omega+k^{2} \Lambda\right)\right]^{-1}$ is the residue of the hydrodynamic pole.

$\Lambda$ is the matrix of transport coefficients. Expression (55) is the discrete analog of the usual Green-Kubo expressions for transport coefficients. Explicitly,

$$
\Lambda(s)=\tau V^{-1} \sum_{t=0}^{\prime} e^{-s t}\langle I \mid I(t)\rangle\langle A \mid A\rangle_{0}^{-1}
$$

where the prime indicates that the $t=0$ term has a relative weight of $1 / 2$. Aside from the sum over discrete times, this is the primary difference in the form of the Green-Kubo relation from that for continuum dynamics [45].

\section{Explicit expressions}

For the current model, the hydrodynamic variables are the density $\delta A_{1}(\mathbf{k})=\delta \rho(\mathbf{k})$, the components of the momentum density $\delta A_{\beta}=\delta v_{\beta-1}$, with $\beta=2, \ldots, d+1$, and the energy density $\delta e(\mathbf{k})$. However, in the following, it is more convenient to work with the variable

$$
\delta A_{d+2}(\mathbf{k})=\frac{1}{2} \sum_{j}\left[v_{j}^{2}-\left\langle v_{j}^{2}\right\rangle\right] e^{i \mathbf{k} \cdot \boldsymbol{\xi}_{j}} f(a \mathbf{k}),
$$

which we will call reduced energy density, instead of the cell energy. If we now assume, as in an ideal gas, that the particle coordinates and velocities are uncorrelated, the susceptibility matrix for this set of variables is diagonal

$$
\left\langle A_{\beta} \mid A_{\gamma}\right\rangle=\rho\left(\begin{array}{ccc}
1 & 0 & 0 \\
0 & k_{B} T \mathbf{I} f^{2}(a \mathbf{k}) & 0 \\
0 & 0 & \frac{d}{2}\left(k_{B} T\right)^{2} f^{2}(a \mathbf{k})
\end{array}\right)
$$

where $\mathbf{I}$ is the $d$-dimensional identity matrix, and $\rho$ is the particle density.

The reduced frequency matrix $\omega$ is defined in Eq. (48). By symmetry, the only nonzero elements of $\omega$ are those coupling the momentum density with either the density or the reduced energy density. For $\left.\mathbf{k} \cdot\left\langle\mathbf{D}_{1} \mid A_{2}\right\rangle\right|_{k=0}$, the correlation of the particle density flux with the momentum density, one finds

$$
\begin{aligned}
\left.\mathbf{k} \cdot\left\langle\mathbf{D}_{1} \mid A_{2}\right\rangle\right|_{k=0} & =-\left.\frac{1}{\tau V} \sum_{j, l} \mathbf{k} \cdot\left\langle\Delta \mathbf{r}_{j} v_{l x} e^{i \mathbf{k} \cdot\left(\boldsymbol{\xi}_{l}-\mathbf{r}_{j}\right)}\right\rangle\right|_{k=0} \\
& =-\rho k_{B} T k_{x}
\end{aligned}
$$

since $\Delta \mathbf{r}_{j}=\tau \mathbf{v}_{j}$. Although we know from symmetry that $\left.\mathbf{k} \cdot\left\langle\mathbf{D}_{1} \mid A_{2}\right\rangle\right|_{k=0}=\left.\mathbf{k} \cdot\left\langle\mathbf{D}_{2} \mid A_{1}\right\rangle\right|_{k=0}$, it is instructive to calculate $\left.\mathbf{k} \cdot\left\langle\mathbf{D}_{2} \mid A_{1}\right\rangle\right|_{k=0}$ explicitly. In particular,

$$
\begin{aligned}
\left.\mathbf{k} \cdot\left\langle\mathbf{D}_{2} \mid A_{1}\right\rangle\right|_{k=0}= & -\frac{1}{\tau V} \sum_{j, l} \mathbf{k} \cdot\left\langle\left\{ v_{j x} \Delta \boldsymbol{\xi}_{j}\right.\right. \\
& \left.\left.+\Delta v_{j x} \Delta \boldsymbol{\xi}_{j}^{s}\right\} e^{i \mathbf{k} \cdot\left(\mathbf{r}_{l}-\boldsymbol{\xi}_{j}\right)}\right\rangle\left.\right|_{k=0} .
\end{aligned}
$$

It can be shown using the techniques described in Sec. II B of Part 2 that the ensemble average on the second term on the right hand side of Eq. (60) is zero. It is also shown in Sec. II B 1 of Part 2 that $\Delta \boldsymbol{\xi}_{j}$ can be replaced by $\tau \mathbf{v}_{j}$ in expectation values of quantities which are linear in $\Delta \boldsymbol{\xi}_{j}$, so that, in agreement with Eq. (59),

$$
\begin{aligned}
\left.\mathbf{k} \cdot\left\langle\mathbf{D}_{2} \mid A_{1}\right\rangle\right|_{k=0} & =-\left.\frac{1}{\tau V} \sum_{j, l} \mathbf{k} \cdot\left\langle v_{j x} \Delta \boldsymbol{\xi}_{j} e^{i \mathbf{k} \cdot\left(\mathbf{r}_{l}-\boldsymbol{\xi}_{j}\right)}\right\rangle\right|_{k=0} \\
& =-\left.\frac{k_{B} T}{V} k_{x} \sum_{j, l}\left\langle e^{i \mathbf{k} \cdot i\left(\mathbf{r}_{l}-\boldsymbol{\xi}_{j}\right)}\right\rangle\right|_{k=0} \\
& =-\rho k_{B} T k_{x}
\end{aligned}
$$

where the last equality follows from $\left.\Sigma_{l}\left\langle e^{i \mathbf{k} \cdot\left(\mathbf{r}_{l}-\boldsymbol{\xi}_{j}\right)}\right\rangle\right|_{k=0}$ $=N \delta_{l j}$. The other terms in the frequency matrix can be evaluated in a similar fashion. The final result is

$$
\boldsymbol{\omega}=-\rho k_{B} T\left(\begin{array}{ccc}
0 & \hat{\mathbf{k}} & 0 \\
\hat{\mathbf{k}} & 0 & k_{B} T \hat{\mathbf{k}} \\
0 & k_{B} T \hat{\mathbf{k}} & 0
\end{array}\right)
$$

The reduced fluxes $I_{\beta}(\hat{\mathbf{k}}, t)$ defined in Eq. (43) are

$$
\begin{gathered}
I_{1}(\hat{\mathbf{k}}, t)=\frac{1}{\tau} \sum_{j} \hat{\mathbf{k}} \cdot\left[-\Delta \mathbf{r}_{j}(t)+\tau \mathbf{v}_{j}(t)\right] \\
I_{1+\beta}(\hat{\mathbf{k}}, t)=\frac{1}{\tau} \sum_{j}\left(-\left[v_{j \beta}(t) \hat{\mathbf{k}} \cdot \Delta \boldsymbol{\xi}_{j}(t)+\Delta v_{j \beta}(t) \hat{\mathbf{k}} \cdot \Delta \xi_{j}^{s}(t)\right]\right. \\
\left.+\frac{\tau \hat{k}_{\beta}}{d} v_{j}^{2}(t)\right)
\end{gathered}
$$

for $\beta=1, \ldots, d$, and 


$$
\begin{aligned}
I_{d+2}(\hat{\mathbf{k}}, t)= & \frac{1}{\tau} \sum_{j}\left(-\left[\left\{v_{j}^{2}(t) / 2-c_{v} T\right\} \hat{\mathbf{k}} \cdot \Delta \boldsymbol{\xi}_{j}(t)\right.\right. \\
& \left.\left.+\frac{1}{2} \Delta v_{j}^{2}(t) \hat{\mathbf{k}} \cdot \Delta \boldsymbol{\xi}_{j}^{\xi}(t)\right]+\tau k_{B} T \hat{\mathbf{k}} \cdot \mathbf{v}_{j}(t)\right),
\end{aligned}
$$

where $c_{v}=d k_{B} / 2$ is the specific heat per particle at constant volume of an ideal gas and $\Delta v_{j}^{2}(t)=v_{j}^{2}(t+\tau)-v_{j}^{2}(t)$. Since $\Delta \mathbf{r}_{i}(t)=\tau \mathbf{v}_{j}(t), I_{1}(\hat{\mathbf{k}}, t)=0$ to this order in $k$.

The hydrodynamic transport coefficients for a simple liquid are the kinematic shear and bulk viscosities $\nu$ and $\gamma$ and the thermal transport coefficient $\lambda_{T}$. Using Eq. (64) in Eq. (56), the asymptotic (long-time limit) shear and bulk viscosities are found to be

$$
\begin{aligned}
& \nu\left(\delta_{\beta \varepsilon}+\frac{d-2}{d} \frac{k_{\beta} k_{\varepsilon}}{k^{2}}\right)+\gamma \frac{k_{\beta} k_{\varepsilon}}{k^{2}} \\
& =\frac{\tau}{N k_{B} T} \sum_{t=0}^{\infty}\left\langle I_{1+\beta}(\hat{\mathbf{k}}, 0) \mid I_{1+\varepsilon}(\hat{\mathbf{k}}, t)\right\rangle .
\end{aligned}
$$

Similarly, the thermal transport coefficient is

$$
\lambda_{T}=\frac{\tau}{c_{v} N k_{B} T^{2}} \sum_{t=0}^{\infty}\left\langle I_{d+2}(\hat{\mathbf{k}}, 0) \mid I_{d+2}(\hat{\mathbf{k}}, t)\right\rangle
$$

$\lambda_{T}$ is related to the thermal diffusivity $D_{T}$ by $D_{T}$ $=\lambda_{T} c_{v} / c_{p}$, where $c_{p}=c_{v}+k_{B}$ is the specific heat per particle at constant pressure. The thermal conductivity, $\kappa_{T}$ is $\kappa_{T}=\rho c_{p} D_{T}$.

\section{NUMERICS}

\section{A. Relaxation towards equilibrium}

A series of simulations were performed on systems with periodic boundary conditions consisting of $L^{2}$ cells, with $L$ ranging from 16 to 64 . The average number of particles per cell, $M$, was between 5 and 70 . The simulations were initiated with a random distribution of particles, with random velocities with components uniformly distributed in the interval $\left[-v_{\max }, v_{\max }\right]$. The velocity distribution was found to quickly relax to a Maxwell-Boltzmann distribution. In order to characterize this relaxation in more detail, we measured the fourth moment of the velocity distribution, $M_{4}$ $=\sum_{j=1}^{N}\left(v_{j x}^{4}+v_{j x}^{4}\right)$, which is a nonconserved quantity, as a function of time. It was found that $M_{4}$ relaxes exponentially to the equilibrium value given by the Maxwell-Boltzmann distribution with a relaxation time $\tau_{R}$ which is essentially temperature independent, see Fig. 2. As can be seen, $\tau_{R}$ is proportional to the average number of particles in a cell, $M$, and depends strongly on the value of the rotation angle $\alpha$. It diverges approximately as $\tau_{R} \sim \alpha^{-2}$ for $\alpha \rightarrow 0$; in this limit, there are no collisions and thermal equilibrium cannot be achieved.

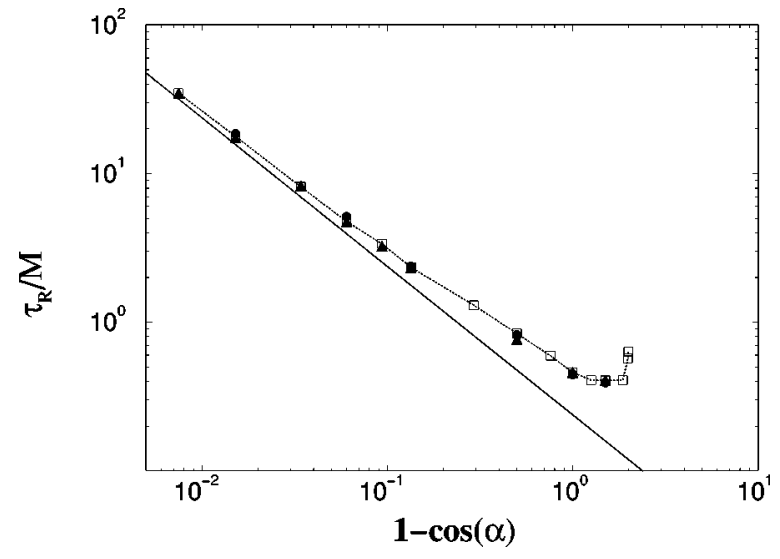

FIG. 2. Normalized relaxation time $\tau_{R} / M$ for the fourth moment of the velocity distribution as a function of $1-\cos (\alpha)$, where $\alpha$ is the rotation angle. Measurements with different values of $M$ (average number of particles per box) fall on the same curve: $M$ $=15$ (filled circles), $M=35$ (squares and dashed line), $M=70$ (filled triangles). The solid line is a plot of $[1-\cos (\alpha)]^{-1}$. Parameters: time step $\tau=1, k_{B} T=0.01275$, and $L=16$.

\section{B. Measuring the shear viscosity}

We present here results for the shear viscosity at nonzero wave vector measured for both short and long times using two different approaches. First, the kinematic viscosity $\nu$ is determined from the temporal decay of the vorticity. Since the decay is quite rapid, this allows us to measure the shorttime viscosity in equilibrium. The viscosity is also determined at long times by measuring the linear response of the fluid to a spatially modulated force. As will be seen, both measurements agree, validating the fluctuation-dissipation theorem for this model. In Part 2, we determine the transport coefficients using Green-Kubo relations for the microscopic stress tensor. This allows us to determine the time-dependent viscosity from an equilibrium measurement, and enabled us to observe long-time tails in two dimensions.

In order to determine the shear viscosity, we need to project out the longitudinal part of the velocity field. We therefore consider the correlations of the vorticity, $w_{k}$ $=k_{x} \tilde{u}_{y}-k_{y} \tilde{u}_{x}$, where $\tilde{u}_{x}$ is the Fourier transform of the $x$ component of the macroscopic velocity. Fluctuating hydrodynamics [46] predicts that vorticity correlations decay as

$$
\left\langle w_{k}(t) w_{-k}(0)\right\rangle \sim \exp \left[-\nu(k) k^{2} t\right] .
$$

We have used Eq. (68) to determine the shear viscosity both with and without the random shift before the stochastic rotation step for the parameters $k_{B} T=0.01275(\lambda / a=0.11), \alpha$ $=90^{\circ}, M=35$, and $L=16$. Without the shift, the value of $\nu$ determined in this way is at least a factor of 2 larger than that given by Eq. (47) of Ref. [31] for $\alpha=90^{\circ}$. We also observed an anomaly in $\nu(\mathbf{k})$ for small mean free path if one component of the wave vector is zero. In particular, as can be seen in Fig. 3 (solid lines), the viscosity at wave vector $\mathbf{k}$ $=2 \pi(1,0) / L$ is approximately four times smaller than for 


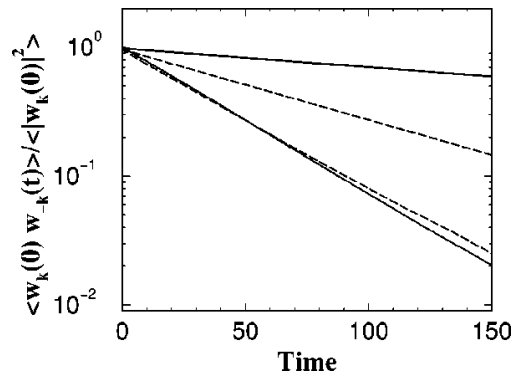

FIG. 3. Normalized vorticity correlations $\left\langle w_{k}(0) w_{-k}(t)\right\rangle /\left\langle\left|w_{k}(0)\right|\right\rangle$ versus time for two different wave vectors $\mathbf{k}$ at small mean free path, $\lambda / a=0.11$, with (dashed lines) and without (solid lines) the random shift of the grid before the collision step. The upper solid and dashed lines are results for $\mathbf{k}$ $=2 \pi(0,1) / L$. The lower solid and dashed lines correspond to $\mathbf{k}$ $=2 \pi(1,1) / L$. Parameters: $k_{B} T=0.01275, \alpha=90^{\circ}, M=35$, and $L=16$.

$\mathbf{k}=2 \pi(1,1) / L$. This would imply, for example, that the viscosity for Poiseuille flow would be four times larger if the flow axis makes an angle of $45^{\circ}$ with respect to the cell axes than if it were parallel. We also observed a similar drastic change in the value of $\nu\left(\mathbf{k}_{0}\right)$ if we imposed a homogeneous flow parallel to the wave vector $\mathbf{k}_{0}=\left(k_{x}, 0\right)$. This breakdown of Galilean invariance for small mean free path is consistent with the anomalous behavior described in Sec. I A for the self-diffusion constant.

If a random shift of the particle coordinates is performed prior to the collision step, as described in Sec. I A, all the anomalies described above disappear. Figure 3 (dashed lines) shows the exponential decay of the vorticity correlations for two different wave vectors when there is a random shift. As can be seen, the viscosity at $\mathbf{k}=2 \pi(1,0) / L$ is now essentially the same as for $\mathbf{k}=2 \pi(1,1) / L$. We also found that $\nu$ no longer depends on the value of a superimposed homogeneous flow in this case. The shifting procedure, in conjunction with the stochastic collision, leads to an additional contribution to the viscosity which removes the $k$-dependent anomalies. This is the source of the additional term in the stress tensor, Eq. (64), which determines the shear viscosity via the Green-Kubo relation (66).

We have also measured the viscosity at long times using a nonequilibrium (linear response) technique in order to validate the fluctuation-dissipation theorem for this model. In a periodic $L \times L$ system, a constant, but spatially modulated force, $\mathbf{f}=\left(f_{x}, 0\right)$, with $f_{x}=F \cos (2 \pi y / L) / M$, was applied to every particle. After a sufficiently long time, the averaged cell velocity in $x$ direction converged to $\left\langle u_{x}\right\rangle=A \cos (k y)$, with wave number $k=2 \pi / L$. The Navier-Stokes equation predicts $A=F /\left(\nu k^{2}\right)$. A was measured for different values of the driving force $F$, extrapolated to $F=0$, and used to determine the $k$-dependent viscosity $\nu(k)$. Note that this method yields the viscosity at large times. In two dimensions, the viscosity is predicted to diverge very slowly (logarithmically) with time, with an amplitude proportional to $1 / M$ (for more details, see Part 2 of this paper). In a finite system, however, $\nu$ will saturate at some system-size dependent value. In our case, the average particle number per box, $M$, was large, and we did not see any significant difference between the viscosities measured using the equilibrium method (via the vorticity) and the nonequilibrium (constant force) method, in agreement with the fluctuation-dissipation theorem.

\section{CONCLUSION}

In this paper, we have presented a detailed, systematic derivation of the equations of motion for the correlation functions and discrete Green-Kubo relations for the stochastic rotation dynamics model originally proposed by Malevanets and Kapral [31,32]. Several results for this model have been presented in Refs. [31,32]; for example, it was shown that there is an $H$ theorem and that this algorithm yields the correct ideal gas hydrodynamics equations. However, the analysis of Refs. [31,32], as well as the applicability of the original algorithm, is limited to situations in which the mean free path is larger than the cell size, so that the assumption of molecular chaos is valid. In this, and a previous paper [33], we have shown that there are unphysical anomalies in the transport coefficients and a breakdown of Galilean invariance for small mean free paths. It was also shown that a small modification of the algorithm, which involves a randomization of the collision environment, resolves these problems. In addition, as will be shown in detail in Part 2 of this series, there are large discrepancies between the value of the viscosity measured in simulations and the result of Malevanets and Kapral (Eq. (47) in Ref. [31] and Eq. (29) in Ref. [32]) in two dimensions for $\alpha \approx 90^{\circ}$. This discrepancy is resolved in Part 2 by explicitly considering the effects of the discrete cell structure, an effect which was neglected in Refs. [31,32].

The discrete-time projection operator formalism employed in this paper is similar to that utilized by Dufty and Ernst [41] in their derivation of the Green-Kubo relations for lattice gas cellular automata. Using this approach, we were able to incorporate the cell shifting procedure discussed in Sec. I A, and it was shown that while the shifting procedure does not change the reversible Euler terms in the equations of motion, it does lead to new contributions to the GreenKubo relations. The Green-Kubo relations are analyzed in Part 2, where accurate, analytical expressions for the various transport coefficients are derived which are valid for all values of $\lambda / a$ and arbitrary Mach number. The derivations presented in Part 2 require no assumptions regarding molecular chaos; they are quite general, and as will be shown elsewhere, can be used to analyze more complicated situations such as binary fluids or nonideal gases.

\section{ACKNOWLEDGMENTS}

Support from the National Science Foundation under Grant Nos. DMR-9712134 and DMR-0083219, and the donors of The Petroleum Research Fund, administered by the ACS, are gratefully acknowledged. T.I. thanks G. Gompper for his hospitality at the IFF, Forschungszentrum Jülich. 
[1] G.A. Bird, Phys. Fluids 13, 2676 (1970).

[2] G. A. Bird, Molecular Gas Dynamics and the Direct Simulation of Gas Flows (Clarendon Press, Oxford, 1994).

[3] P.J. Hoogerbrugge and J.M.V.A. Koelman, Europhys. Lett. 19, 155 (1992).

[4] J.M.V.A. Koelman and P.J. Hoogerbrugge, Europhys. Lett. 21, 369 (1993).

[5] P. Español, Phys. Rev. E 52, 1734 (1995).

[6] C. Marsh, G. Backx, and M.H. Ernst, Europhys. Lett. 38, 411 (1997); C. Marsh, G. Backx, and M.H. Ernst, Phys. Rev. E 56, 1976 (1997).

[7] C.A. Marsh and Y.M. Yeomans, Europhys. Lett. 37, 511 (1997).

[8] E.S. Boek, P.V. Coveney, H.N.W. Lekkerkerker, and P. van der Schoot, Phys. Rev. E 55, 3124 (1997).

[9] E.S. Boek, P.V. Coveney, and H.N.W. Lekkerkerker, J. Phys.: Condens. Matter 8, 9509 (1997).

[10] A.G. Schlijper, P.J. Hoogerbrugge, and C.W. Manke, J. Rheol. 39, 567 (1995).

[11] Y. Kong, C.M. Manke, W.G. Madden, and A.G. Schlijper, Baltzer. Tribology Lett. 3, 133 (1997).

[12] K.E. Novik and P.V. Coveney, Int. J. Mod. Phys. C 8, 909 (1997)

[13] S.I. Jury, P. Bladon, S. Krishna, and M.E. Cates, Phys. Rev. E 59, R2535 (1999).

[14] W. Dzwinel and D. Yuen, Int. J. Mod. Phys. C 11, 1 (2000).

[15] P. Español, Europhys. Lett. 40, 631 (1997).

[16] J. Bonet Avalós and M. Mackie, Europhys. Lett. 40, 141 (1997).

[17] M. Ripoll, P. Español, and M.H. Ernst, Int. J. Mod. Phys. C 9, 1329 (1999).

[18] M. Ripoll, M.H. Ernst, and P. Español, J. Chem. Phys. 115, 7271 (2001).

[19] F.J. Alexander and A.L. Garcia, Comput. Phys. 11, 588 (1997).

[20] E.P. Muntz, Annu. Rev. Fluid Mech. 21, 387 (1989).

[21] A. L. Garcia, Numerical Methods for Physics (Prentice-Hall, Englewood, Cliffs, NJ, 2000).

[22] E. Salomons and M. Mareschal, Phys. Rev. Lett. 69, 269 (1992).
[23] D.L. Morris, L. Hannon, and A.L. Garcia, Phys. Rev. A 46, 5279 (1992).

[24] E.S. Oran, C.K. Oh, and B.Z. Cybyk, Annu. Rev. Fluid Mech. 30, 403 (1998).

[25] G.A. Bird, Comput. Math. Appl. 35, 1 (1998).

[26] C. Cercignani, Rarefied Gas Dynamics (Cambridge University Press, Cambridge, 2000).

[27] F.J. Alexander, A.L. Garcia, and B.J. Alder, Phys. Rev. Lett. 74, 5212 (1995).

[28] J.M. Montanero and A. Santos, Phys. Rev. E 54, 438 (1996).

[29] J.M. Montanero and A. Santos, Phys. Fluids 9, 2057 (1997).

[30] F.J. Alexander, A.L. Garcia, and B.J. Alder, Physica A 240, 196 (1997).

[31] A. Malevanets and R. Kapral, J. Chem. Phys. 110, 8605 (1999).

[32] A. Malevanets and R. Kapral, J. Chem. Phys. 112, 7260 (2000).

[33] T. Ihle and D.M. Kroll, Phys. Rev. E 63, 020201(R) (2001).

[34] A. Lamura, G. Gompper, T. Ihle, and D.M. Kroll, Europhys. Lett. 56, 319 (2001).

[35] E. Allahyarov and G. Gompper, Phys. Rev. E 66, 036702 (2002).

[36] T. Ihle and D. M. Kroll (unpublished).

[37] S. Succi, I.V. Karlin, and H. Chen, Rev. Mod. Phys. 74, 1203 (2002).

[38] C.A. Marsh and P.V. Coveney, J. Phys. A 31, 6561 (1998).

[39] U. Frisch, D. d'Humières, B. Hasslacher, P. Lallemand, Y. Pomeau, and J.-P. Rivet, Complex Syst. 1, 649 (1987).

[40] F. Reif, Fundamentals of Statistical and Thermal Physics (McGraw-Hill, New York, 1965).

[41] J.W. Dufty and M.H. Ernst, J. Phys. Chem. 93, 7015 (1989).

[42] R. Zwanzig, in Lectures in Theoretical Physics (Wiley, New York, 1961), Vol. 3, p. 135.

[43] H. Mori, Prog. Theor. Phys. 33, 423 (1965).

[44] H. Mori, Prog. Theor. Phys. 34, 399 (1965).

[45] D. Forster, Hydrodynamic Fluctuations, Broken Symmetry, and Correlation Functions (Benjamin, Reading, MA, 1975).

[46] L. D. Landau and E. M. Lifshitz, Fluid Dynamics (Pergamon Press, Oxford, 1959). Note that the chapter dealing with fluctuating hydrodynamics is absent in later editions. 